\title{
MODELLING OF SPECIAL EQUIPMENT SUPERVISION GAME CONSIDERING RISK EXPECTATION
}

\author{
Huang, J.-H. ; He, S. ; Chen, Y.** \& Yang, C.-H. *, \\ * School of Economics \& Management, Fuzhou University, Fuzhou, China \\ ${ }^{* *}$ Sino-US Global Logistics Institute, Shanghai Jiaotong University, Shanghai, China \\ E-Mail: hcysun@fzu.edu.cn ( ${ }^{\#}$ Corresponding author)
}

\begin{abstract}
To reveal the causes of unlawful behaviours between special equipment quality supervision institutions and third-party special equipment inspection institutions, an evolutionary game model was developed. Since the expected payoff matrix frequently used in general game model cannot accurately describe the influence of the risk expectations, the prospect value function was proposed to build the revenue perception matrix of the model. Then, the evolutionary characteristics were analysed through replicator dynamics equations. Accordingly, the evolutionary stable strategies were obtained based on the Jacobian matrix. Moreover, the dynamic evolution phase diagrams were formulated to describe the behaviours. Finally, numerical simulation examples were conducted to investigate the evolutionary path and the evolutionarily stable strategies. Results show that the probability of the third-party special equipment inspection institution choosing the passive performance strategy is negatively correlated with the expected risk value. The supervision probability of the quality supervision institution is directly proportional to the social benefits. In addition, the supervision probability is dependent on the penalty risks, and the initial population scale.

(Received in May 2017, accepted in October 2017. This paper was with the authors 2 months for 1 revision.)
\end{abstract}

Key Words: Prospect Theory, Third-Party Special Equipment Inspection Institution, Supervision Strategies, Evolutionary Game

\section{INTRODUCTION}

Special equipment can pose threats to the safety of life and property, such as boilers, pressure vessels, pressure pipelines, elevators, passenger ropeways, and large-scale recreational facilities. Most countries have set up the specialized quality supervision institutions to guarantee the special equipment's quality safety. However, accidents resulting from quality safety problems of special equipment still occur frequently, such as the explosion of Tosco Avon, an oil refinery of Martinez in California, US in 1997, the explosion of liquefied petroleum gas cylinders of Xi'an Coal and Gas Co., Ltd. in Shanxi Province, China in March 1998, and the explosion of the P-Xylene Project in Zhangzhou, Fujian, China in 2015. In reality, special equipment inspection is a difficult task, which requires professional skills, and knowledge systems. Quality inspection institutions with limited professional assets and techniques have difficulties in perfectly accomplishing the inspection task. Therefore, in developed countries, such as the United States of America, Japan, Germany, and the UK, third-party inspection institutions are mostly entrusted for special equipment inspection, whereas the quality supervision institutions are mainly responsible for ensuring institutional construction, business permit, qualification certification, and supervision. The independent third-party inspection institutions play a significant role in special equipment quality safety inspections, which has become not only a consensus but also an irresistible development trend of the field [1].

In practice, the third-party inspection institutions are driven by interests to illegally gain profits and exhibit rent-seeking behaviours (i.e., hiding and falsifying inspection results), which result in the invalidity of inspection results [2-4]. For a long time, the improvement of the supervision system targeted at the third-party inspection institutions is regarded as an 
effective approach to ensuring the authenticity and effectiveness of inspection results. For example, a suitable contract is designed to reduce the probability of corruption and dereliction of duties of the third-party inspection institutions. Previous research mainly focused on demonstrating the unlawful facts found in third-party inspection institutions and discussing the measures to reduce the occurrence of unlawful events. However, the essential causes behind the problem have been ignored [5]. In addition, the motivations of unlawful activities of third-party inspection institutions have a close bearing not only on illegal gains but also on risk expectations. The expected utility theory used in the classical game model is unsuitable for analysing the impact of the psychological expectation of third-party inspection institutions on the analytical outcomes.

According to the above analysis, this study examines a game problem of supervision between third-party inspection institutions and special equipment quality supervision institutions. The evolutionary game model is developed to reveal the reasons of unlawful behaviours among the third-party inspection institutions. In this model, the prospect theory is applied to describe the risk expectations.

The rest of this study is organized as follows. In Section 2, the related literature was discussed, and the differences between this study and the previous research work were analysed. In Section 3, the evolutionary game model was formulated and the evolutionarily stable solutions were analysed. In Section 4, numerical simulations were conducted to obtain the evolutionary path and the evolutionarily stable strategies. Section 5 concludes this study with future research directions.

\section{STATE OF THE ART}

The studies related to the inspection of third-party inspection institutions have been studied from the following three perspectives:

First, the defects were analysed when supervision institutions is the subjects of quality inspection. For instance, Mol pointed out that supervision institutions serving as the subjects of quality inspection cannot guarantee the authenticity and reliability of inspection in China [6]. Liu et al. conducted an empirical analysis of risk factors influencing the quality of dairy products using the binary choice model, discovering that the role of supervision institutions in controlling and restricting melamine contamination is limited [7]. Through case analysis or empirical analysis, it was found that the problems of quality inspection results mainly originated from the malposition of inspection subjects. However, the reasons behind the malposition of inspection subjects were not analysed deeply in these studies.

Second, the necessity of third-party inspection institutions was discussed. For instance, Hatanaka et al. affirmed that the emergence of third-party inspections holds positive significance toward producers, distributors, and other organizations [8]. Dranove and Jin observed that the intervention of third-party inspection institutions can effectively alleviate the problem of information asymmetry and increase consumers' trust for inspection results [9]. Strausz substantiated the effectiveness of the work through third-party quality inspection institutions by building the principal-agent mechanism [10]. These studies emphasized the fairness of the third-party inspection institutions and their role as a bridge. However, the new conflicts and the issue of interest game, which might be triggered due to the introduction of third-party inspection institutions, have been neglected.

Third, it was discussed how to effectively monitor third-party inspection institutions under the game environment. The third-party inspection institutions, tempted by illegal gains, can be involved in a game relationship with supervision departments resulting to falsification and hiding of results [11]. For instance, Liu et al. developed a game model between governmental supervision institution and the coal mine industry and analysed the influence of supervision 
intensity on behaviours of the coal mine industry [12]. Considering information loss, and the bounded rationality of game players, Yi and Yang, and Sartakhti et al. asserted that an evolutionary game model is highly suitable for the description and analysis of issues of the kind, respectively $[13,14]$. Strausz analysed the effectiveness of supervision, and verified that the supervision institutions' tightening of penalty for unlawful behaviours can improve supervision effects [10]. However, these studies focused on one-way supervision by supervising institutions, and ignored potential countermeasures adopted by supervised targets against the tightened penalty. In addition, Zhang found that the supervision effects of inspection institutions on third-party inspection units can influence equipment users' psychological expectations about equipment safety [15]. Yu and Liu studied the game problem of supervision between the supervision institution and the third-party inspection institution, and pointed out that the third-party institution has psychological expectations of its unlawful risks. Moreover, the expected risks can influence strategy choice of the game players [16]. In these studies, the expected utility theory was applied to the game revenue functions and the strategies were chosen based on the expected revenue rather than the expected risks.

To sum up, the aforementioned studies mainly adopted the authorization mechanism, the principal-agent mechanism, and the trust game model as research methods, with cooperation and trust as research hypotheses. Most research outcomes emphasized the advantages of the third-party inspection institutions in eliminating the information asymmetry, promoting cooperation, and ensuring the fairness of inspection results. However, the nature of the thirdparty inspection institutions to pursue their self-interest and the potential conflict between third-party inspection institutions and supervision institutions were neglected. Some studies pointed out that the utility of risks expected by the third-party inspection institutions can influence their strategy choice. Nevertheless, the efforts are not made about how to describe the risk expectation of game players and how to analyse the influence of risk expectation on game outcomes.

The prospect theory may be preferable in describing risk expectation and perceived value [17-19]. Thus, the prospect value function is considered to represent the expected revenue of players. Accordingly, an evolutionary game model was formulated between the quality supervision institution and third-party special equipment inspection institution to discuss the influence of risk expectations on the evolutionary paths and evolution stabilization strategies.

\section{METHODOLOGY}

\subsection{Problem description and hypotheses}

To formulate the game of supervision between the quality supervision institution and the third-party special equipment inspection institution, the assumptions are listed as follows:

Assumption 1: The quality supervision and third-party inspection institutions are the game players with bounded rationality. Their strategy choices are provided based on their payoffs using the different strategies rather than the utility of strategies themselves. According to prospect theory proposed by Kahneman and Tversky, the risk decision-making process is composed of the editing and evaluation phases [20]. In the evaluation phase, the expected utility $(V)$ is decided by the utility function $(v)$ and the weighting function $(\pi)$, where

$$
\begin{gathered}
V(p, x)=\sum_{i=1}^{n} \pi\left(p_{i}\right) v\left(\Delta w_{i}\right) \\
v\left(\Delta w_{i}\right)= \begin{cases}\Delta w_{i}^{\varepsilon} & x \geq 0 \\
-\lambda\left(-\Delta w_{i}\right)^{\eta} & x<0\end{cases}
\end{gathered}
$$




$$
\pi^{-}\left(p_{i}\right)=\frac{p_{i}^{\rho}}{\left[p_{i}^{\rho}+\left(1-p_{i}\right)^{\rho}\right]^{\frac{1}{\rho}}}, \pi^{+}(p)=\frac{p_{i}^{\sigma}}{\left[p_{i}^{\sigma}+\left(1-p_{i}\right)^{\sigma}\right]^{\frac{1}{\sigma}}}
$$

In Eq. (1), $p_{i}$ denotes the probability that event $i$ happens; $\Delta w_{i}$ is the losses or gains of the decision-maker with respect to the reference point when event $i$ happens, namely, $\Delta w_{i}=w_{i}-w_{0}$. The utility function, $v\left(\Delta w_{i}\right)$, is the function of gains or losses $\left(\Delta w_{i}\right)$ of event $i$, with respect to the reference point, and it satisfies the following conditions: when $\Delta w_{i}>0, v\left(\Delta w_{i}\right)$ is a concave function; when $\Delta w_{i}<0, v\left(\Delta w_{i}\right)$ is a convex function, and the sensitivity of the convex function is stronger than that of the concave function. The weight function $\pi\left(p_{i}\right)$ is the probability function concerning the occurrence of event $i$, and $\pi(0)=0, \pi(1)=1 \cdot \pi^{-}\left(p_{i}\right)$ is the weighting function when the decision-maker is faced with losses, whereas $\pi^{+}\left(p_{i}\right)$ is the weighting function when the decision-maker is faced with gains.

Assumption 2: The strategy profile of the quality supervision institution is active supervision, $H_{1}$; passive supervision, $H_{2}$. The population proportion of choosing the active and negative strategies is $x$ and $1-x$, respectively, where $0 \leq x \leq 1$. The strategy profile of the third-party inspection institution is the active performance of inspection responsibilities, $S_{1}$; the passive performance of inspection responsibilities, $\left.S_{2}\right\}$. The population proportion of choosing the active and negative strategies is $y$ and $1-y$, respectively, where $0 \leq y \leq 1$. Both $x$ and $y$ are the functions of time $t$.

Assumption 3: When the strategy profile is $\left\{H_{1}, S_{1}\right\}$, the quality safety level of the special equipment is the highest. Suppose the safety risk cost under the condition is 0 . When the strategy profile of the two game players is $\left\{H_{1}, S_{2}\right\}$ or $\left\{H_{2}, S_{1}\right\}$, the quality safety level of the is lower than the case $\left\{H_{1}, S_{1}\right\}$. When the strategy profile of the two game players is $\left\{H_{2}, S_{2}\right\}$, the safety risk level is the highest.

Assumption 4: When problems with the special equipment quality emerge, the two game players should assume the corresponding amount of the cost of risk. If the cost of risk assumed by the third-party institution is $L$, then the cost of risk assumed by the quality supervision institution can be formulated as $k L$, where $k$ is the risk transfer coefficient.

\subsection{Model interpretation}

A typical special equipment supervision process can be illustrated as shown in Fig. 1. This study focuses on the game problem between the quality supervision institution and the thirdparty inspection institution.

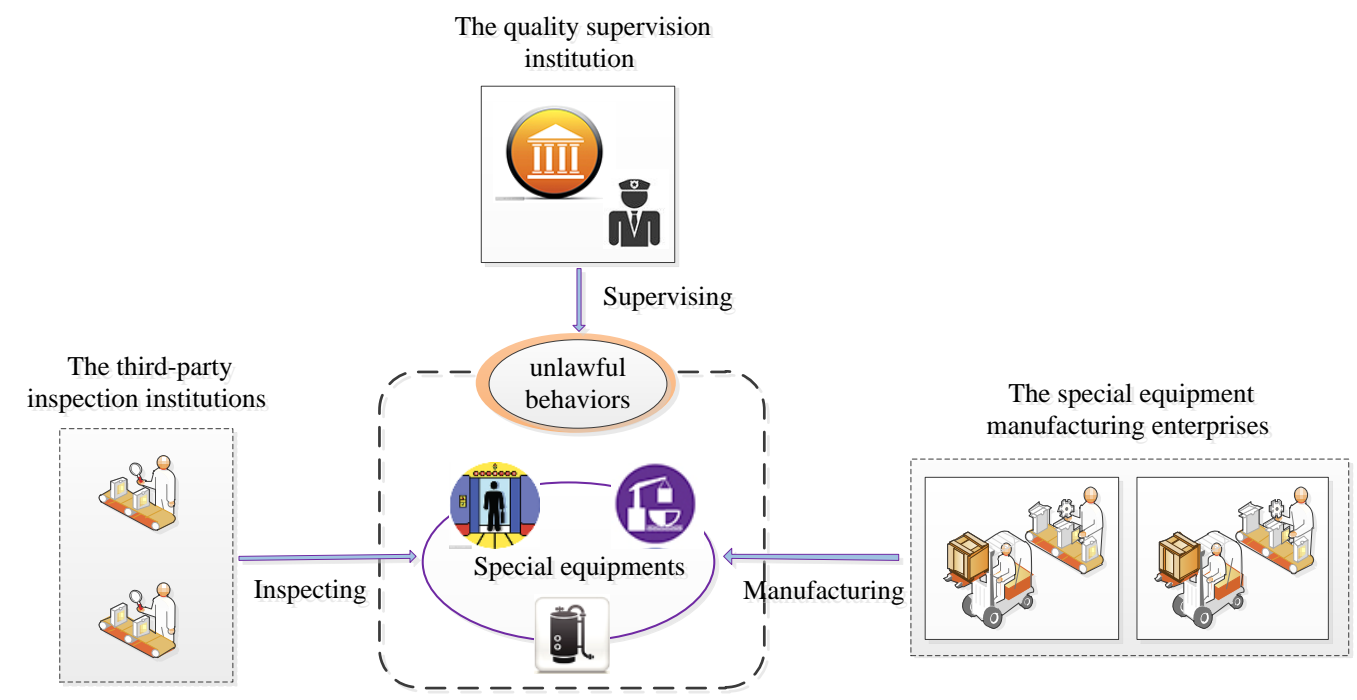

Figure 1: Typical special equipment supervision process. 
Based on the aforementioned assumptions, the payoff matrix concerning the game model between the quality supervision institution and the third-party inspection institution are presented as shown in Table I. The objectives of two players are to seek an evolutionarily stable solution.

Table I: Payoff matrix of the game model.

\begin{tabular}{|l|c|c|}
\hline \multirow{2}{*}{$\begin{array}{c}\text { Quality supervision } \\
\text { institution }\end{array}$} & \multicolumn{2}{|c|}{ Third-party inspection institution } \\
\cline { 2 - 3 } & $\begin{array}{c}\text { Active performance of inspection } \\
\text { responsibilities }\left(S_{1}\right)\end{array}$ & $\begin{array}{c}\text { Negative performance of inspection } \\
\text { responsibilities }\left(S_{2}\right)\end{array}$ \\
\hline Active supervision $\left(H_{1}\right)$ & $R_{g}-C_{g}, B_{t}-C_{t}$ & $R_{g}+L_{t 1}-C_{g}-k e_{2} L_{t 2}, B_{t}-L_{t 1}-e_{2} L_{t 2}$ \\
Passive supervision $\left(H_{2}\right)$ & $-k e_{1} L_{t 2}, B_{t}-C_{t}-e_{1} L_{t 2}$ & $-k L_{t 2}, B_{t}-L_{t 2}$ \\
\hline
\end{tabular}

The parameters in Table I are explained as follows:

$R_{g}$ - The rewards obtained by the quality supervision institution from active supervision, including the recognition of the public about the quality supervision institution. $R_{g} \in[0,+\infty)$.

$C_{g}$ - The cost that the quality supervision institution paid for active supervision, which can be regarded as the perceived value of all supervision efforts. $C_{g} \in[0,+\infty)$.

$B_{t}$ - The fees paid by the quality supervision institution for entrusting the third-party inspection institution for the special equipment quality inspection. $B_{t} \in[0,+\infty)$.

$C_{t}$ - The extra cost paid by the third-party inspection institution for active performance of its responsibilities. $C_{t} \in[0,+\infty)$.

$L_{t 1}$ - The penalties are imposed on the third-party inspection institution for the passive performance of its inspection responsibilities. $L_{t 1} \in[0,+\infty)$.

$L_{t 2}$ - The special equipment quality risk cost carried by the third-party inspection institution. $L_{t 2} \in[0,+\infty)$.

$e_{1}$ - The discount coefficient of the special equipment quality risk cost borne by the two players when their strategy profile is $\left\{H_{2}, S_{1}\right\} . e_{1} \in[0,1]$.

$e_{2}$ - The discount coefficient of the special equipment quality risk cost borne by the two players when their strategy profile is $\left\{H_{1}, S_{2}\right\} . e_{2} \in[0,1]$.

(1) When the strategy choice of the two players constitutes the strategy profile, $\left\{H_{1}, S_{1}\right\}$, $C_{g}$ can be defined as follows by using Eq. (1):

$$
C_{g}=\pi\left(1-P_{1}\right) v\left(c_{g}\right)+\pi\left(P_{1}\right) v(0)=\pi(1) v\left(c_{g}\right)+\pi(0) v(0),
$$

where $\pi(0)=0, \pi(1)=1$ [hence, $C_{g}=v\left(c_{g}\right)$ ] and $c_{g}$ is the cost of time paid by the quality supervision institution. Similarly, $C_{t}$ can be defined as follows:

$$
C_{t}=\pi\left(1-P_{1}\right) v\left(c_{t}\right)+\pi\left(P_{1}\right) v(0)=v\left(c_{t}\right),
$$

where $c_{t}$ is the extra cost paid by the third-party inspection institution for the adoption of the active strategy.

(2) When the strategy choice of the two game players constitutes the strategy profile, $\left\{H_{2}, S_{2}\right\}$, based on Assumption 4 and Assumption 1, $L_{t 2}$ can be formulated as follows:

$$
L_{t 2}=\pi\left(P_{2}\right) v\left(l_{t 2}\right)+\pi\left(1-P_{2}\right) v(0)=\pi\left(P_{2}\right) v\left(l_{t 2}\right) .
$$

(3) When the strategy choice of the two game players constitutes the strategy profile, $\left\{H_{1}, S_{2}\right\}, L_{t 1}$ can be formulated as follows:

$$
L_{t 1}=\pi\left(P_{3}\right) v\left(l_{t 1}\right)+\pi\left(1-P_{3}\right) v(0)=\pi\left(P_{3}\right) v\left(l_{t 1}\right) .
$$

(4) When the strategy choices of the two game players constitute the strategy profile, $\left\{H_{2}, S_{1}\right\}$, we can deduce that the third-party inspection institution, in addition to paying the extra cost, $\left(B_{t}-C_{t}\right)$, for the active performance of inspection responsibilities, the third-party inspection institution should also undertake the perceived safety risk, $e_{1} L_{t 2}$. The quality 
supervision institution should bear the risk cost, $k e_{1} L_{t 2}$, transferred from the third-party inspection institution.

\subsection{Model construction and analysis}

Based on the replicator dynamics equations which were proposed by Friedman [21], the expected utility of the quality supervision institution in choosing the active strategy and the passive strategy are $U_{H 1}$ and $U_{H 2}$, respectively, and the respective average potential utility, $\bar{U}_{H}$, are formulated as follows:

$$
\left\{\begin{array}{l}
U_{H 1}=\left(R_{g}-C_{g}\right) y+\left(R_{g}+L_{t 1}-C_{g}-k e_{2} L_{t 2}\right)(1-y) \\
U_{H 2}=-k e_{1} L_{t 2} y-k L_{t 2}(1-y) \\
\bar{U}_{H}=x U_{H 1}+(1-x) U_{H 2}
\end{array}\right.
$$

Similarly, the expected utilities of the third-party inspection institution under the two strategies $S_{1}$ and $S_{2}$ are $U_{S 1}$ and $U_{S 2}$, respectively, and the respective average potential utility, $\bar{U}_{S}$, are formulated as follows:

$$
\left\{\begin{array}{l}
U_{S 1}=\left(B_{t}-C_{t}\right) x+\left(B_{t}-C_{t}-e_{1} L_{t 2}\right)(1-x) \\
U_{S 2}=\left(B_{t}-L_{t 1}-e_{2} L_{t 2}\right) x+\left(B_{t}-L_{t 2}\right)(1-x) \\
\bar{U}_{S}=y U_{S 1}+(1-y) U_{S 2}
\end{array}\right.
$$

The replicator dynamic function of $x$ and $y$ can be obtained through the asymmetric replicator dynamic evolution:

$$
\begin{aligned}
& F(x)=\frac{d x}{d z}=x(1-x)\left[R_{g}-C_{g}+\left(L_{t 1}-k e_{2} L_{t 2}+k L_{t 2}\right)(1-y)+k e_{1} L_{t 2} y\right] \\
& G(y)=\frac{d y}{d x}=y(1-y)\left[L_{t 1} x+\left(e_{1}+e_{2}-1\right) L_{t 2} x-\left(e_{1}-1\right) L_{t 2}-C_{t}\right]
\end{aligned}
$$

Eq. (6) shows that, when $x=0,1$ or

$$
y=\frac{C_{g}-R_{g}+\left(e_{2}-1\right) k L_{t 2}-L_{t 1}}{\left(e_{1}+e_{2}-1\right) k L_{t 2}-L_{t 1}}
$$

the proportion of the quality supervision institution in choosing the active supervision is locally stable. Eq. (7) indicates that, when and only when $y=0,1$ or

$$
x=\frac{C_{t}+\left(e_{1}-1\right) L_{t 2}}{L_{t 1}+\left(e_{1}+e_{2}-1\right) L_{t 2}}
$$

the proportion of the third-party institution in choosing the active performance of inspection responsibilities is locally stable. Therefore, the equation set formed by Eqs. (6) and (7) has five locally stable points, including $O(0,0), A(1,0), B(0,1), C(1,1)$ and

$$
D\left(\frac{C_{t}+\left(e_{1}-1\right) L_{t 2}}{L_{t 1}+\left(e_{1}+e_{2}-1\right) L_{t 2}}, \frac{C_{g}-R_{g}+\left(e_{2}-1\right) k L_{t 2}-L_{t 1}}{\left(e_{1}+e_{2}-1\right) k L_{t 2}-L_{t 1}}\right)
$$

The evolutionarily stable strategies are solved on the basis of the Jacobian matrix for local stability analysis.

$$
J=\left[\begin{array}{lc}
(1-2 x)\left[R_{g}-C_{g}+y\left(k e_{2} L_{t 2}+k e_{1} L_{t 2}-L_{t 1}-k L_{t 2}\right)+k L_{t 2}\left(1-e_{2}\right)+L_{t 1}\right] & x(1-x)\left(k e_{2} L_{t 2}+k e_{1} L_{t 2}-L_{t 1}-k L_{t 2}\right) \\
y(1-y)\left[L_{t 1}+\left(e_{1}+e_{2}-1\right) L_{t 2}\right] & (1-2 y)\left[L_{t 1} x+\left(e_{1}+e_{2}-1\right) L_{t 2} x-\left(e_{1}-1\right) L_{t 2}-C_{t}\right]
\end{array}\right]
$$

The matrix determinant can be written as follows: 


$$
\begin{aligned}
& \operatorname{det} J=(1-2 x)(1-2 y)\left[R_{g}-C_{g}+y\left(k e_{2} L_{t 2}+k e_{1} L_{t 2}-L_{t 1}-k L_{t 2}\right)+k L_{t 2}\left(1-e_{2}\right)+L_{t 1}\right] \\
& {\left[L_{t 1} x+\left(e_{1}+e_{2}-1\right) L_{t 2} x-\left(e_{1}-1\right) L_{t 2}-C_{t}\right]+x y(1-x)(1-y)\left(k e_{2} L_{t 2}+k e_{1} L_{t 2}-L_{t 1}-k L_{t 2}\right)} \\
& {\left[L_{t 1}+\left(e_{1}+e_{2}-1\right) L_{t 2}\right]}
\end{aligned}
$$

The matrix trace can be written as follows:

$$
\begin{aligned}
& \operatorname{trJ}=(1-2 x)\left[R_{g}-C_{g}+y\left(k e_{2} L_{t 2}+k e_{1} L_{t 2}-L_{t 1}-k L_{t 2}\right)+k L_{t 2}\left(1-e_{2}\right)+L_{t 1}\right]+(1-2 y)\left[L_{t 1} x+\right. \\
& \left.\left(e_{1}+e_{2}-1\right) L_{t 2} x-\left(e_{1}-1\right) L_{t 2}-C_{t}\right]
\end{aligned}
$$

When the following four conditions are substantiated, the two players will both choose the active strategy. Thereafter, the model converges at $C(1,1)$.

$$
\left\{\begin{array}{l}
C_{g} \leq R_{g}+k L_{t 2}\left(1-e_{2}\right)+L_{t 1}, C_{g} \leq R_{g}+k e_{1} L_{t 2} \\
C_{t} \leq L_{t 1}+e_{2} L_{t 2}, C_{t} \leq L_{t 2}-e_{1} L_{t 2}
\end{array}\right.
$$

The analytical results of five stable points, namely, $O(0,0), A(1,0), B(0,1), C(1,1)$ and $D$, are presented in Table II.

Table II: Local stability analytical results of four stable equilibria.

\begin{tabular}{|c|l|c|c|c|c|}
\hline $\begin{array}{c}\text { Equilibrium } \\
\text { point }\end{array}$ & \multicolumn{1}{|c|}{$\operatorname{det} J$} & Sign & trJ & Sign & Classification \\
\hline \multirow{2}{*}{$O(0,0)$} & {$\left[R_{g}-C_{g}+k L_{t 2}\left(1-e_{2}\right)+\right.$} & + & {$\left[R_{g}-C_{g}+k L_{t 2}\left(1-e_{2}\right)+\right.$} & + & Unstable \\
& $\left.L_{t 1}\right]\left[-\left(e_{1}-1\right) L_{t 2}-C_{t}\right]$ & & $\left.L_{t 1}\right]\left[-\left(e_{1}-1\right) L_{t 2}-C_{t}\right]$ & & point \\
& $-\left[R_{g}-C_{g}+k L_{t 2}\left(1-e_{2}\right)\right.$ & - & $-\left[R_{g}-C_{g}+k L_{t 2}\left(1-e_{2}\right)\right.$ & Not & Saddle point \\
$A(1,0)$ & $\left.+L_{t 1}\right]\left(L_{t 1}+e_{2} L_{t 2}-C_{t}\right)$ & & $\left.+L_{t 1}\right]+\left(L_{t 1}+e_{2} L_{t 2}-C_{t}\right)$ & fixed & \\
& $-\left[R_{g}-C_{g}+k e_{1} L_{t 2}\right]\left(L_{t 2}\right.$ & - & $-\left[R_{g}-C_{g}+k e_{1} L_{t 2}\right]+\left(L_{t 2}\right.$ & Not & Saddle point \\
$B(0,1)$ & $\left.-e_{1} L_{t 2}-C_{t}\right)$ & & $\left.-e_{1} L_{t 2}-C_{t}\right)$ & \\
& $\left(R_{g}-C_{g}+k e_{1} L_{t 2}\right)\left(L_{t 1}+\right.$ & + & $-\left(R_{g}-C_{g}+k e_{1} L_{t 2}\right)-\left(L_{t 1}+\right.$ & - & ESS \\
$C(1,1)$ & $\left.e_{2} L_{t 2}-C_{t}\right)$ & & $\left.e_{2} L_{t 2}-C_{t}\right)$ & \\
\hline
\end{tabular}

The Jacobian matrix cannot effectively analyse the stability of $D\left(\frac{C_{t}+\left(e_{1}-1\right) L_{t 2}}{L_{t 1}+\left(e_{1}+e_{2}-1\right) L_{t 2}}, \frac{C_{g}-R_{g}+\left(e_{2}-1\right) k L_{t 2}-L_{t 1}}{\left(e_{1}+e_{2}-1\right) k L_{t 2}-L_{t 1}}\right)$. Hence, the differential analysis is conducted. Eqs. (6) and (7) are used to work out the differentials of $y$ and $x$. Thereafter, the differential obtained is substituted with $D$ to obtain the following:

$$
\begin{gathered}
\frac{d F}{d y}=\frac{\left(C_{t}+e_{1} L_{t 2}-L_{t 2}\right)\left(L_{t 1}+e_{2} L_{t 2}-C_{t}\right)}{\left[L_{t 1}+\left(e_{1}+e_{2}-1\right) L_{t 2}\right]^{2}} \cdot\left[\left(e_{1}+e_{2}-1\right) k L_{t 2}-L_{t 1}\right] \\
\frac{d G}{d x}=\frac{\left(R_{g}-C_{g}+e_{1} k L_{t 2}\right)}{\left[\left(e_{1}+e_{2}-1\right) k L_{t 2}-L_{t 1}\right]^{2}} \cdot\left[L_{t 1}+\left(e_{1}+e_{2}-1\right) L_{t 2}\right]
\end{gathered}
$$

Given $x, y \in[0,1]$, for $D\left(\frac{C_{t}+\left(e_{1}-1\right) L_{t 2}}{L_{t 1}+\left(e_{1}+e_{2}-1\right) L_{t 2}}, \frac{C_{g}-R_{g}+\left(e_{2}-1\right) k L_{t 2}-L_{t 1}}{\left(e_{1}+e_{2}-1\right) k L_{t 2}-L_{t 1}}\right)$ to make sense, then the following condition must be satisfied:

$$
0 \leq \frac{C_{t}+\left(e_{1}-1\right) L_{t 2}}{L_{t 1}+\left(e_{1}+e_{2}-1\right) L_{t 2}} \leq 1,0 \leq \frac{C_{g}-R_{g}+\left(e_{2}-1\right) k L_{t 2}-L_{t 1}}{\left(e_{1}+e_{2}-1\right) k L_{t 2}-L_{t 1}} \leq 1
$$


Given that the equation set converges at four conditions of $C(1,1)$, the calculated results of Eqs. (9) and (10) are, hence, positive or, in other words, point $D$ is an unstable point.

To sum up, the dynamic evolution phase diagram of the game model can be obtained as shown in Fig. 2.

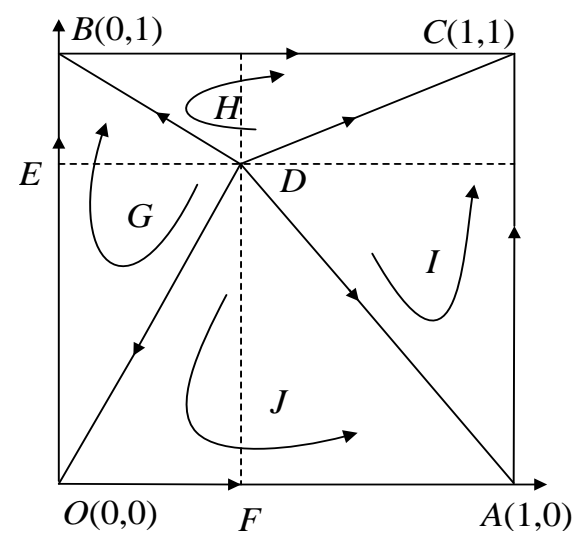

Figure 2: Equilibrium dynamic evolution phase diagram.

\section{RESULT ANALYSIS AND DISCUSSIONS}

In this section, we aim to conduct our analytical results based on the real industry cases, Chongqing Municipal Quality and Technology Supervision Bureau in China are taken as a case study (Data source: http://203.93.109.253/Item/1773.aspx). Based on the "Chongqing Municipal Elevator Safety Management Methods", the penalty for the violation of the special equipment inspection rules is $3.5 \mathrm{~K} \$$, i.e., $L_{t 1}=3.5 \mathrm{~K} \$$; the cost of the quality supervision institution generated by its active supervision is $1.6 \mathrm{~K} \$$, i.e., $C_{g}=1.6 \mathrm{~K} \$$; the social benefits of proper supervision are set to be $7.8 \mathrm{~K} \$$, i.e., $R_{g}=7.8 \mathrm{~K} \$$; the extra cost paid by the third-party inspection institution for the active performance of inspection responsibilities is $2 \mathrm{~K} \$$, i.e., $C_{t}=2 \mathrm{~K} \$$; and the special equipment quality safety risk cost carried by the third-party special equipment inspection institution for its violation of inspection rules is $15 \mathrm{~K} \$$, i.e., $L_{t 2}=15 \mathrm{~K} \$$. When a special equipment quality safety event happens, the risk transfer coefficient of the quality supervision institution is 1 , i.e., $k=1$. The discount coefficients of the special equipment quality safety risk cost are set to be 0.4 and 0.6 , respectively, i.e., $e_{1}=0.4$ and $e_{2}=0.6$.

\subsection{Influence of the initial value changes on evolution outcomes}

The replicator dynamic functions of the quality supervision institution and the third-party inspection institution are represented by $d x / d t$ and $d y / d t$, respectively. When the initial population scale, $x_{0}$, of the quality supervision institution choosing active supervision is set to be $0.2,0.4,0.6$ and 0.8 , and $y_{0}=0.3$, the evolutionarily stable strategy stabilizes at $y^{*}=0$ (Fig. $3)$.

The initial value of the third-party inspection institution choosing the strategy of "active performance of inspection responsibilities" is set to be $0.2,0.4,0.6$ and 0.8 , and $x_{0}=0.3$. When the initial population scale of the third-party inspection institution reaches $y_{0}=0.8$, the evolutionarily stable strategy of the quality inspection institution changes from $x^{*}=1$ to $x^{*}=0$ (Fig. 4). Therefore, a responsible inspection institution can contribute to alleviating the work pressure of supervision institution. In sum, the following conclusion can be reached:

Conclusion 1: Along with the increasing population scale of the third-party inspection institution choosing the "active performance of inspection responsibilities", the quality supervision institution tends to choose the strategy of passive supervision. 


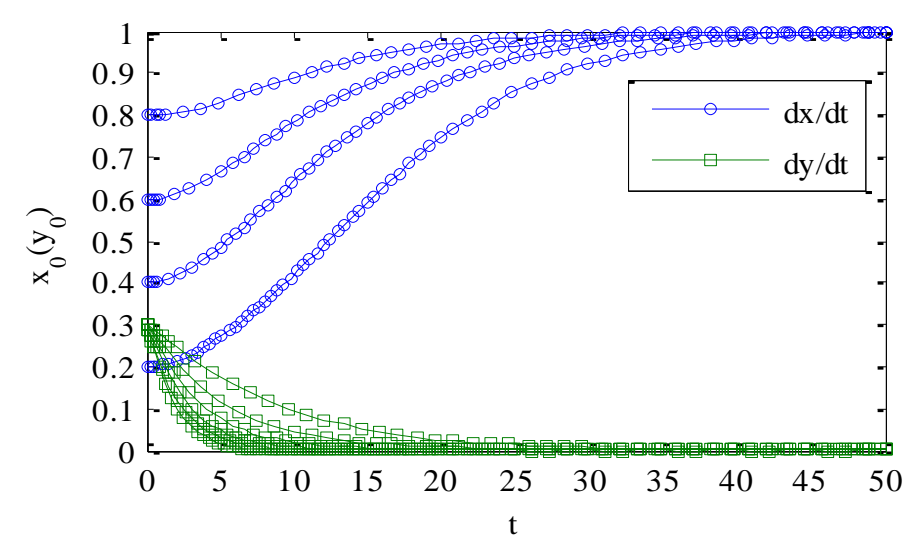

Figure 3: Evolutionarily stable strategy when the initial value $y_{0}=0.3$, whereas $x_{0}$ varies.

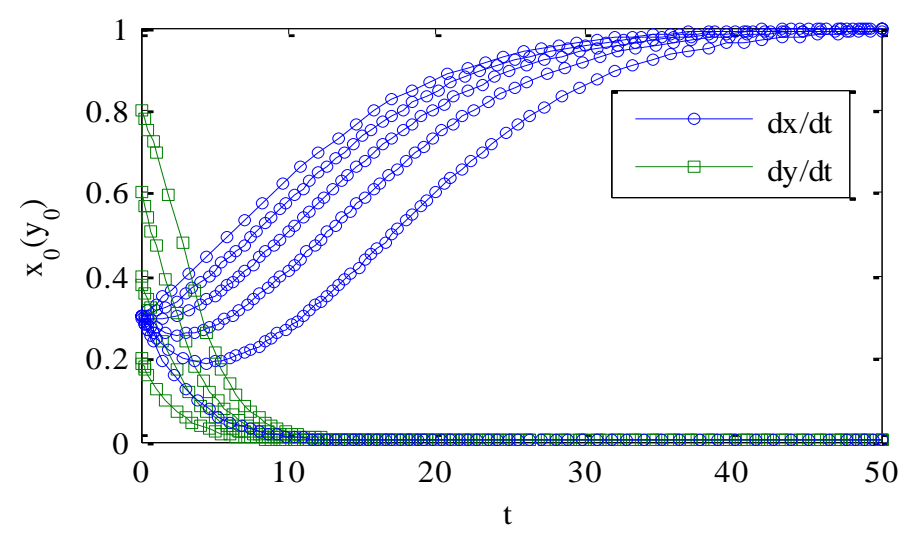

Figure 4: Evolutionarily stable strategy when the initial value $x_{0}=0.3$, whereas $y_{0}$ varies.

\subsection{Influence of changes of the penalty risk perception, $L_{t 1}$, on evolution outcomes}

To analyse the influence of different penalty risk perceptions on evolutionary game outcomes between the quality supervision institution and the third-party inspection institution, $L_{t 1}$ is set to be 3.5, 5.6, 6.4, 7.2, 7.8, and 9.

Fig. 5 exhibits that, when $L_{t 1}=3.5$ and $L_{t 1}=5.6$, the evolutionarily stable strategy stabilizes at $x^{*}=1$. When $L_{t 1}=6.4$, the evolutionarily stable strategy tends to be $x^{*}=0$. A critical value exists in the section of 5.6-6.4. When $L_{t 1}$ is smaller than the critical value, $x$ converges at 1 . Therefore, when the third-party inspection institution has a low risk perception of penalty from the quality supervision institution, the quality supervision institution is prone to the strategy of active supervision. When $L_{t 1}$ is larger than the critical value, $x$ converges at 1 . Presently, the high penalty cost pushes the quality supervision institution to choose the strategy of passive supervision, but the pace of evolution is slow.

Fig. 6 shows that a critical value exists in the section of 5.6-6.4. When $L_{t 1}$ exceeds the critical value, $y$ immediately converges at 1 . Meanwhile, along with the increasing of $L_{t 1}$, the convergence of $y$ speeds up. The perception of a high penalty cost forces the third-party inspection institution to choose the strategy of the active performance of inspection responsibilities. This scenario suggests that the setting of a high penalty amount by the quality supervision institution can prompt the third-party inspection institution to pay considerable attention to perform its responsibilities. Overall, the following conclusion can be summarized:

Conclusion 2: The risk perception of different penalty amounts has a significant influence on the evolutionarily stable strategy of the quality supervision and third-party inspection institutions. 


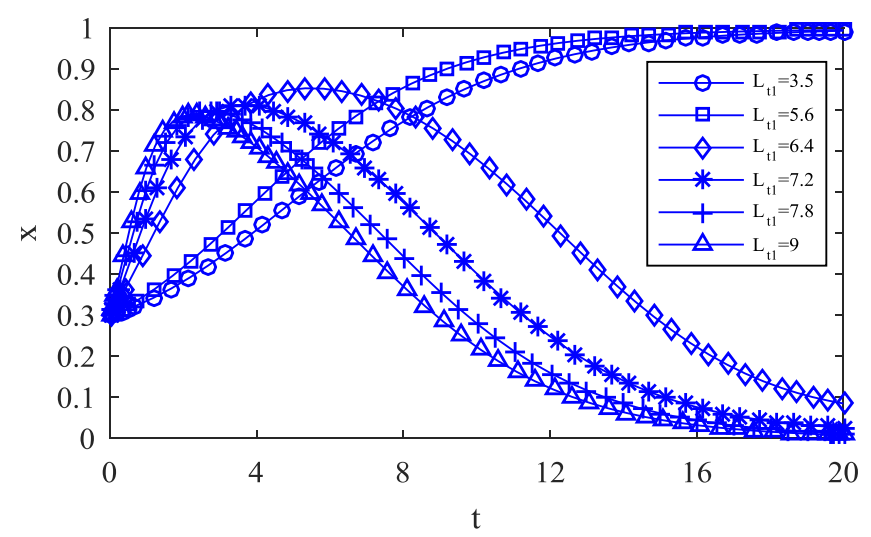

Figure 5: Influence of the penalty risk perceptions on the evolutionarily stable strategy of the quality supervision institution.

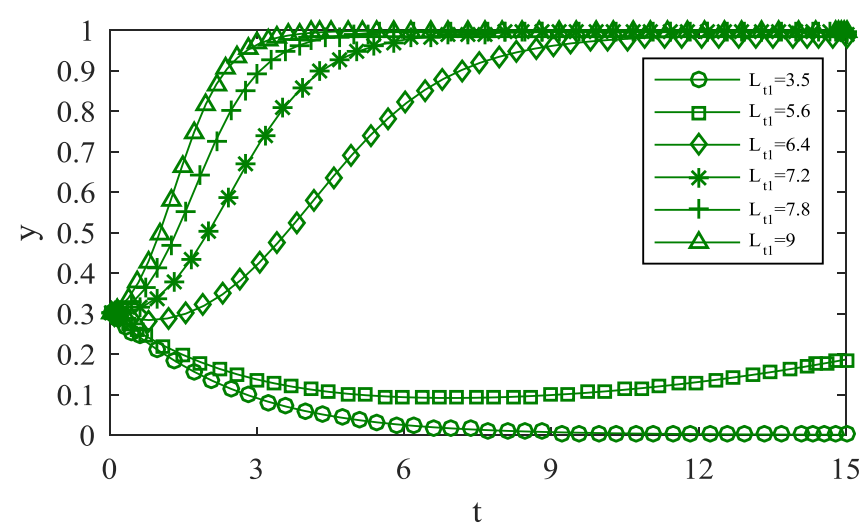

Figure 6: Influence of the penalty risk perceptions on the evolutionarily stable strategy of the thirdparty inspection institution.

\subsection{Influence of changes of quality risk perception, $L_{t 2}$, on evolution outcomes}

Fig. 7 shows that the evolutionary path and the evolution process of the quality supervision institution differ under different perceived values of safety risks (when the perceived value of special equipment quality safety risk, $L_{t 2}$, is set to be $15,20,24,30,35$, and 40 ), but the population scale of the quality supervision institution choosing positive supervision stabilizes at $x^{*}=1$.

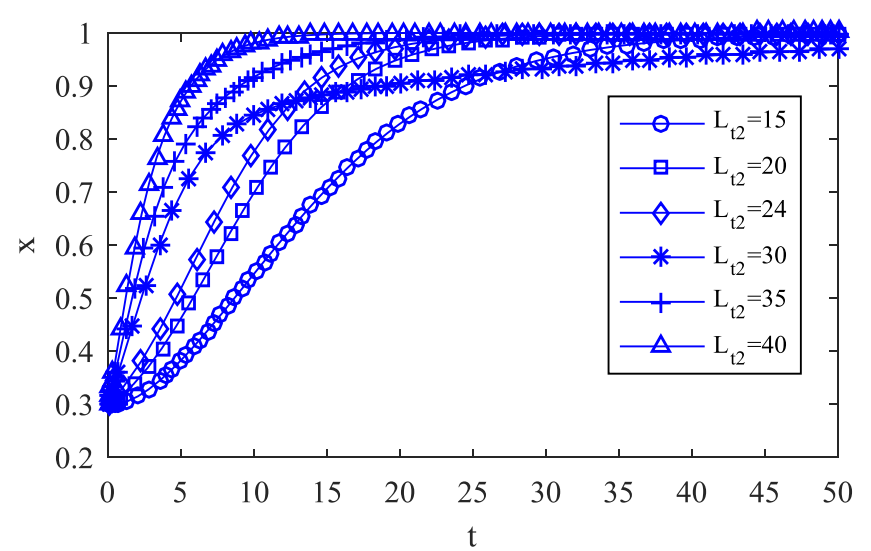

Figure 7: Influence of the different perceived values of special equipment quality safety risk on the evolutionarily stable strategy of the quality supervision institution.

Fig. 8 exhibits that, when $L_{t 2}$ is set to be 15 and 20, the evolution curves almost overlap with another one. When $L_{t 2}=24$, the evolutionarily stable strategy of the third-party inspection 
institution finally stabilizes at $y^{*}=1$, and the convergence accelerates along with the increasing of $L_{t 2}$. So, when a third-party inspection institution is faced with a major special equipment quality safety risk, this institution prefers to adopt the strategy of active supervision. In sum, the following conclusion can be obtained:

Conclusion 3: Changes of $L_{t 2}$ has no influence on the strategy choice of the quality supervision institution. Along with the increase of the perceived quality safety risk value, the third-party inspection institution tends to choose the active strategy.

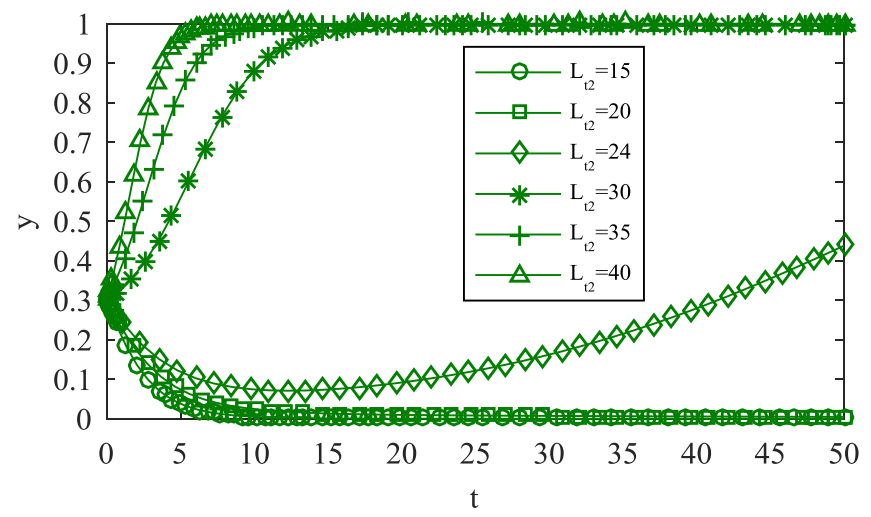

Figure 8: Influence of the different perceived values of special equipment quality safety risk on the evolutionarily stable strategy of the third-party inspection institution.

\section{CONCLUSIONS}

To identify the causes of the unlawful behaviours of the special equipment inspection institutions, the risk expectations were accurately described based on the prospect value function and the evolutionary game model were developed. Finally, the evolutionary path and the evolutionarily stable strategies were discussed. The following conclusions were obtained:

(1) The prospect value function formulated based on the prospect theory can effectively describe the influence of expected risks on strategy choices of the game players. When the expected risks value of third-party inspection institution is greater than a certain value, the third-party institution is prone to choose the active strategy. Otherwise, the passive strategy is preferred to adopt.

(2) The supervision strategy choice of the quality supervision institution is correlated with the special equipment quality safety risk. When the special equipment quality safety risk is small, the quality supervision institution tends to choose the strategy of passive supervision.

(3) Both the evolutionary path and the evolutionarily stable strategy (ESS) of game are obviously influenced by the quality safety risk perceptions of the quality supervision institution and the penalty risk perceptions of the third-party inspection institution.

As a public service department, the quality supervision institution's supervision revenue includes not only the economic benefits but also social benefits. However, in this study the supervision revenue is simplified and defined by a variable parameter. How to effectively evaluate the social benefits of supervision should be considered in future research.

\section{ACKNOWLEDGEMENTS}

This study was supported by the National Social Science Foundation of China (Grant No. 13BGL059), MOE (Ministry of Education in China) Project of Humanities and Social Sciences (Grant No. 17YJC630194), and the Fujian Provincial Social Science Foundation (Grant No. FJ2016B059). 


\section{REFERENCES}

[1] De Maeyer, P.; Estelami, H. (2011). Consumer perceptions of third party product quality ratings, Journal of Business Research, Vol. 64, No. 10, 1067-1073, doi:10.1016/j.jbusres.2011.02.001

[2] Albersmeier, F.; Schulze, H.; Jahn, G.; Spiller, A. (2009). The reliability of third-party certification in the food chain: From checklists to risk-oriented auditing, Food Control, Vol. 20, No. 10, 927-935, doi:10.1016/j.foodcont.2009.01.010

[3] Jahn, G.; Schramm, M.; Spiller, A. (2005). The reliability of certification: Quality labels as a consumer policy tool, Journal of Consumer Policy, Vol. 28, No. 1, 53-73, doi:10.1007/s10603004-7298-6

[4] Rahman, D. M. (2012). But who will monitor the monitor?, American Economic Review, Vol. 102, No. 6, 2767-2797, doi:10.1257/aer.102.6.2767

[5] McAllister, L. K. (2012). Regulation by third-party verification, Boston College Law Review, Vol. 53, No. 1, Paper 1, 1-64

[6] Mol, A. P. J. (2014). Governing China's food quality through transparency: A review, Food Control, Vol. 43, 49-56, doi:10.1016/j.foodcont.2014.02.034

[7] Liu, C. Q.; Sun, Y. Y.; Long, W. J.; Bai, Y. (2009). Competition, management and regulation: An empirical analysis of the influencing factors of melamine contamination in dairy products, Management World, Vol. 25, No. 12, 67-78

[8] Hatanaka, M.; Bain, C.; Busch, L. (2005). Third-party certification in the global agrifood system, Food Policy, Vol. 30, No. 3, 354-369, doi:10.1016/j.foodpol.2005.05.006

[9] Dranove, D.; Jin, G. Z. (2010). Quality disclosure and certification: Theory and practice, Journal of Economic Literature, Vol. 48, No. 4, 935-963, doi:10.1257/jel.48.4.935

[10] Strausz, R. (1997). Delegation of monitoring in a principal-agent relationship, The Review of Economic Studies, Vol. 64, No. 3, 337-357, doi:10.2307/2971717

[11] Celik, G. (2009). Mechanism design with collusive supervision, Journal of Economic Theory, Vol. 144, No. 1, 69-95, doi:10.1016/j.jet.2008.02.006

[12] Liu, D. H.; Xiao, X. Z.; Li, H. Y.; Wang, W. G. (2015). Historical evolution and benefit-cost explanation of periodical fluctuation in coal mine safety supervision: An evolutionary game analysis framework, European Journal of Operational Research, Vol. 243, No. 3, 974-984, doi:10.1016/j.ejor.2014.12.046

[13] Yi, Y.; Yang, H. (2017). Wholesale pricing and evolutionary stable strategies of retailers under network externality, European Journal of Operational Research, Vol. 259, No. 1, 37-47, doi:10.1016/j.ejor.2016.09.014

[14] Sartakhti, J. S.; Manshaei, M. H.; Sadeghi, M. (2017). MMP-TIMP interactions in cancer invasion: An evolutionary game-theoretical framework, Journal of Theoretical Biology, Vol. 412, 17-26, doi:10.1016/j.jtbi.2016.09.019

[15] Zhang, J. H. (2013). Analysis of quality safety game on special equipment, Journal of MacroQuality Research, Vol. 1, No. 2, 75-81, doi:10.3969/j.issn.2095-607X.2013.02.010

[16] Yu, T.; Liu, C. Y. (2016). The analysis of evolution game model and simulation between governments and the third-party in product quality regulation, Chinese Journal of Management Science, Vol. 24, No. 6, 90-96, doi:10.16381/j.cnki.issn1003-207x.2016.06.011

[17] Abdellaoui, M.; Bleichrodt, H.; Paraschiv, C. (2007). Loss aversion under prospect theory: A parameter-free measurement, Management Science, Vol. 53, No. 10, 1659-1674, doi:10.1287/ mnsc. 1070.0711

[18] Zank, H. (2001). Cumulative prospect theory for parametric and multiattribute utilities, Mathematics of Operations Research, Vol. 26, No. 1, 67-81, doi:10.1287/moor.26.1.67.10598

[19] Bleichrodt, H.; Pinto, J. L.; Wakker, P. P. (2001). Making descriptive use of prospect theory to improve the prescriptive use of expected utility, Management Science, Vol. 47, No. 11, 14981514, doi:10.1287/mnsc.47.11.1498.10248

[20] Kahneman, D.; Tversky, A. (1979). Prospect theory: An analysis of decision under risk, Econometrica, Vol. 47, No. 2, 263-292, doi:10.2307/1914185

[21] Friedman, D. (1998). On economic applications of evolutionary game theory, Journal of Evolutionary Economics, Vol. 8, No. 1, 15-43, doi:10.1007/s001910050054 